\section{Different Measurements of Body Fatness and Cardiovascular Risk in Community Dwelling Old Adults}

\author{
Olof G. Geirsdottir ${ }^{1,2^{\star}}$, Milan Chang ${ }^{2,3}$, Palmi V. Jonsson ${ }^{2,4}$, Inga Thorsdottir ${ }^{1}$ and Alfons Ramel ${ }^{1,2}$ \\ ${ }^{1}$ Faculty of Food Science and Nutrition, University of Iceland, Reykjavik, Iceland \\ ${ }^{2}$ The Icelandic Gerontological Research Centre, University Hospital, Faculty of Medicine, University of Iceland, Reykjavik, Iceland \\ ${ }^{3}$ School of Education, University of Iceland, Reykjavik, Iceland \\ ${ }^{4}$ Department of Geriatrics, National University Hospital, Faculty of Medicine, University of Iceland, Reykjavik, Iceland
}

*Corresponding author: Olof Gudny Geirsdottir, Faculty of Food Science and Nutrition, University of Iceland, Tungata 26, 101 Reykjavik, Iceland, Tel: +354 543 9898; E-mail: ogg@hi.is

Received date: July 03, 2018; Accepted date: September 10, 2018; Published date: September 17, 2018

Copyright: (c) 2018 Geirsdottir OG, et al. This is an open-access article distributed under the terms of the Creative Commons Attribution License, which permits unrestricted use, distribution, and reproduction in any medium, provided the original author and source are credited.

\begin{abstract}
Body mass index (BMI) does not necessarily predict cardiovascular disease in old populations which has been called obesity paradox. The aims of the present study were to investigate 1) the associations between BMI and body fat in old adults, and 2) whether more sophisticated measures of body fat are stronger associated with cardiovascular risk than BMI.

In the current cross-sectional analysis, the participants'( $\mathrm{N}=232,65-92$ years) body fatness was estimated using $\mathrm{BMI}$, waist-circumference, DXA (fat mass, visceral fat mass) and cardiovascular risk factors (blood lipids, glucose metabolism, blood pressure) were assessed.

BMI correlated highly with fat mass measured by DXA independently from age tertiles and in both genders. In general, body fatness was positively related to several cardiovascular risk factors and this was persistently observed for all the different measures of body fatness. However, fat measures were not associated with total cholesterol or LDL. Sensitivity analysis indicated that BMI $\geq 25$ identifies $70-100 \%$ of participants with the above mentioned cardiovascular risk factors outside normal range.
\end{abstract}

We conclude in BMI is highly correlated with body fatness in old Icelandic adults. DXA measurement of body fatness is not better in estimating cardiovascular risk in old adults compared to simple BMI.

Keywords: Cross-sectional study; Cardiovascular risk; Body composition; Aging; Body mass index

Abbreviations: hdl: HDL cholesterol; tg: triglycerides; glucose: fasting glucose; insulin: fasting insulin; hbalc: glycosilated hemoglobin; dia: diastolic blood pressure; sys: systolic blood pressure

\section{Introduction}

Obesity is a major non-communicable condition which is one of the main causes of poor health, disability and death worldwide and accounts for considerable health-care expenditures [1]. More specifically, obesity has been linked to diseases like, e.g., cardiovascular disease [2], some cancers [3], type 2 diabetes [4], non-alcoholic fatty liver disease [5] and gastrointestinal disorders [6]. The World Health Organization (WHO) reports that the prevalence of obesity has approximately doubled within the last four decades. Currently, close to two billion adults are overweight [7].

The body mass index (BMI) has been frequently used in epidemiological studies as an estimate for body fatness and individually for the assessment of overweight or obesity $[8,9]$. According to longitudinal studies BMI predicts cardiovascular risk in younger and middle aged populations [8], however this relationships gets blurry in old adults [10] or patient groups [11] and in these groups higher BMI was even associated to lower morbidity and mortality known as obesity paradox. Several explanations have been proposed for this discrepancy [12]. BMI cannot distinguish between lean body mass and fat mass, tissues that can affect disease risk differently [8]. Due to changes in body composition during ageing BMI might be rather an estimate for lean body mass than for fat mass in old adults $[13,14]$. Further on, BMI does not distinguish between metabolically healthy and metabolically unhealthy [15]. Of upmost importance for health consequences of obesity is body fat distribution, which BMI does not account for [16].

Considering the above information, it is reasonable to speculate that more exact but also more expensive measurements of body fat, e.g., dual-energy X-ray absorptiometry (DXA), which can even quantify visceral fat separately, relate better to cardiovascular risk than simple BMI measurements. Thus, the aim of the current investigation was to find out the associations between different estimates of body fat (BMI, DXA) and cardiovascular risk factors in community dwelling old adults. We hypothesized that 1) correlations between BMI and DXA decreases between age quartiles, and 2) that DXA measurements, in particular measurements of visceral fat associate more to risk factors than BMI. This was a secondary data analysis in which we use baseline data from a previously published intervention study [17] in a cross sectional manner. 


\section{Methods and Materials}

\section{Subjects}

The subjects $(\mathrm{N}=232)$ were at least 65 years of age (range $65-92$ years) and we used advertisements posted in the Reykjavik area to recruit them. The following exclusion criteria were used to identify potential subjects: low cognitive function (Mini-Mental State Examination (MMSE) $<19$ points) [18], major orthopedic disease, pharmacological interventions with exogenous testosterone, other drugs or disorder known to affect lean body mass. Recruited participants were apparently healthy, although several had hypertension, hyperlipidemia or type 2 diabetes. The Icelandic National Ethical Committee (VSNb2008060007/03-15) approved the current study and all participants gave their informed written consent for study participation.

\section{Study design}

The data for the current analysis were baseline measurements from the subjects who participated in a 12-week resistance exercise intervention program described and published earlier [15]. No formal sample size calculations were conducted for the present analysis.

\section{Body composition}

Body composition was measured using dual energy x-ray absorptiometer (DXA) from Hologic QDR-2000 plus ${ }^{\circledR}$, Hologic Inc., Waltham, MA, USA (APEX 2.3 software) conducted at the Icelandic Heart Association, Kopavogur, Iceland. Body weight (BW) was measured in light underwear on a calibrated scale (model no. 708, Seca, Hamburg, Germany) and height was measured with a calibrated stadiometer (model no. 206; Seca, Hamburg, Germany). From these measurements BMI was calculated $(\mathrm{kg} / \mathrm{m} 2)$. Waist circumference was taken between the top of the lateral iliac crest and the lowest rib.

\section{Blood pressure measurements}

Blood pressure (BP) was measured according to the user's guide of the blood pressure device (Medisan). After a few minutes rest, the right arm was used and two readings were taken with two minutes inbetween, and the average of those readings was used for statistical analysis.

\section{Blood analyses}

Participants were asked neither to exercise nor to drink alcohol the day before blood sampling. The blood samples stored at $-80^{\circ} \mathrm{C}$ for further analyses which were conducted at the Landspitali-University Hospital in Reykjavik, Iceland which provided also the appropriate reference values.

Total cholesterol (TC), plasma triglycerides (TG) and glucose were analyzed using an enzymatic colori-metric assay and an automated analyzer (Hitachi 911; Roche Diagnostics, Indianapolis, USA). High density lipoprotein (HDL) was determined using polyethylene glycolmodified enzymes and dextran sulfate. Low density lipoprotein (LDL) was estimated using the Friedewald formula. Insulin was measured with electro chemiluminescence immunoassay (ECLIA) on a Modular Analytics E170 system from Roche Diagnostics (Manheim, Germany). HbAlc was measured using a chromatographic-spectrophotometric assay.

\section{Statistical analyses}

SPSS statistical package, version 22.0 (SPSS, Chicago, IL, USA) was used for statistical calculations. The data were checked for normality using the Kolmogorov-Smirnov test and are presented as means \pm standard deviations (SD). Correlations between variables were assessed using the Pearson correlation coefficient $r$. In order to investigate the associations between body fatness and risk factors, linear models with adjustment for age were constructed.

ROC curves were used to calculate the sensitivity of BMI $\geq 25$ to identify participants with cardiovascular risk factor outside the normal range. The significance level was set at $\mathrm{P} \leq 0.05$.

\section{Results}

In Table 1 the participants' characteristics can be seen. Of male participants, only $14.6 \%$ were normal weight, but $38.6 \%$ were overweight and $46.8 \%$ were obese according to BMI. Of female participants, only $27.2 \%$ were normal weight, but $43.4 \%$ were overweight and $29.4 \%$ were obese.

\begin{tabular}{|l|l|l|l|}
\hline & All (N = 232) & male $(\mathbf{n}=\mathbf{9 6})$ & female (n =136) \\
\cline { 2 - 4 } & Mean \pm SD & mean \pm SD & mean \pm SD \\
\hline Age (years) & $73.6 \pm 5.7$ & $74.6 \pm 5.9$ & $72.9 \pm 5.5$ \\
\hline Medication (count) & $2.1 \pm 1.5$ & $2.3 \pm 1.4$ & $1.9 \pm 1.6$ \\
\hline $\begin{array}{l}\text { Waist circumference } \\
\text { (cm) }\end{array}$ & $99.8 \pm 14.4$ & $108.5 \pm 12$ & $93.6 \pm 12.7$ \\
\hline BMl (kg/m2) & $28.8 \pm 4.8$ & $29.7 \pm 4.6$ & $28.1 \pm 4.9$ \\
\hline Lean mass (kg) & $47.8 \pm 10$ & $57.2 \pm 7.6$ & $41 \pm 4.7$ \\
\hline Fat mass (kg) & $31.6 \pm 10.1$ & $32.2 \pm 10.4$ & $31.1 \pm 9.9$ \\
\hline Fat mass (\%) & $38.2 \pm 7.3$ & $33.8 \pm 5.9$ & $41.3 \pm 6.5$ \\
\hline Visceral fat (kg) & $3.3 \pm 1.4$ & $3.8 \pm 1.5$ & $2.9 \pm 1.3$ \\
\hline $\begin{array}{l}\text { Total cholesterol } \\
\text { (mg/dl) }\end{array}$ & $215 \pm 46$ & $197 \pm 43$ & $229 \pm 44$ \\
\hline LDL (mg/dl) & $134 \pm 42$ & $123 \pm 39$ & $142 \pm 43$ \\
\hline HDL (mg/dl) & $60 \pm 17$ & $50 \pm 12$ & $66 \pm 17$ \\
\hline Triglycerides (mg/dl) & $110 \pm 60$ & $117 \pm 73$ & $105 \pm 48$ \\
\hline Glucose (mg/dl) & $89.7 \pm 18.4$ & $94.1 \pm 20.5$ & $86.5 \pm 16.1$ \\
\hline Insulin (mU/ml) & $12 \pm 10.8$ & $14.5 \pm 13.6$ & $10.3 \pm 8$ \\
\hline HbA1c (\%) & $5.7 \pm 0.6$ & $5.8 \pm 0.7$ & $5.6 \pm 0.5$ \\
\hline DBP (mm Hg) & $75.8 \pm 9.4$ & $77.1 \pm 9.2$ & $74.8 \pm 9.5$ \\
\hline SBP (mm Hg) & $144.8 \pm 19.2$ & $151.8 \pm 18.5$ & $139.8 \pm 18.3$ \\
\hline
\end{tabular}

Table 1: Characteristics of the participants.

Table 2 shows correlations between BMI and other estimates of body fat grouped by gender and age-tertiles. BMI was significantly correlated to all other fat measurements with mostly very similar and high correlation coefficients in both genders and all three age groups. 
Citation: Geirsdottir OG, Chang M, Jonsson PV, Thorsdottir I, Ramel A (2018) Different Measurements of Body Fatness and Cardiovascular Risk in Community Dwelling Old Adults. J Nutr Disorders Ther 8: 236. doi:10.4172/2161-0509.1000236

Page 3 of 5

\begin{tabular}{|c|c|c|c|c|c|c|c|}
\hline & & \multicolumn{3}{|l|}{ male } & \multicolumn{3}{|l|}{ female } \\
\hline & & tertile 1 & tertile 2 & tertile 3 & tertile 1 & tertile 2 & tertile 3 \\
\hline & & $(n=27)$ & $(n=36)$ & $(n=33)$ & $(n=65)$ & $(n=37)$ & $(n=34)$ \\
\hline \multirow{2}{*}{ Waist circumference $(\mathrm{cm})$} & $r$ & 0.844 & 0.906 & 0.882 & 0.862 & 0.883 & 0.835 \\
\hline & P-value & $<0.001$ & $<0.001$ & $<0.001$ & $<0.001$ & $<0.001$ & $<0.001$ \\
\hline \multirow{2}{*}{ Body fat (\%) } & $r$ & 0.737 & 0.782 & 0.863 & 0.796 & 0.823 & 0.373 \\
\hline & P-value & $<0.001$ & $<0.001$ & $<0.001$ & $<0.001$ & $<0.001$ & 0.03 \\
\hline \multirow{2}{*}{ Fat mass $(\mathrm{kg})$} & $r$ & 0.845 & 0.897 & 0.923 & 0.922 & 0.926 & 0.774 \\
\hline & P-value & $<0.001$ & $<0.001$ & $<0.001$ & $<0.001$ & $<0.001$ & $<0.001$ \\
\hline \multirow{2}{*}{ Visceral fat $(\mathrm{kg})$} & r & 0.863 & 0.871 & 0.92 & 0.874 & 0.915 & 0.685 \\
\hline & P-value & $<0.001$ & $<0.001$ & $<0.001$ & $<0.001$ & $<0.001$ & $<0.001$ \\
\hline
\end{tabular}

Table 2: Correlation^ between BMI and other estimates of body fat in age tertiles.

Figures 1 and 2 are based on the linear models and show the associations between body fat measurements and cardiovascular risk factors. These calculations were done separately for both genders and corrected for age. Additional correction for drug use did not change the results.

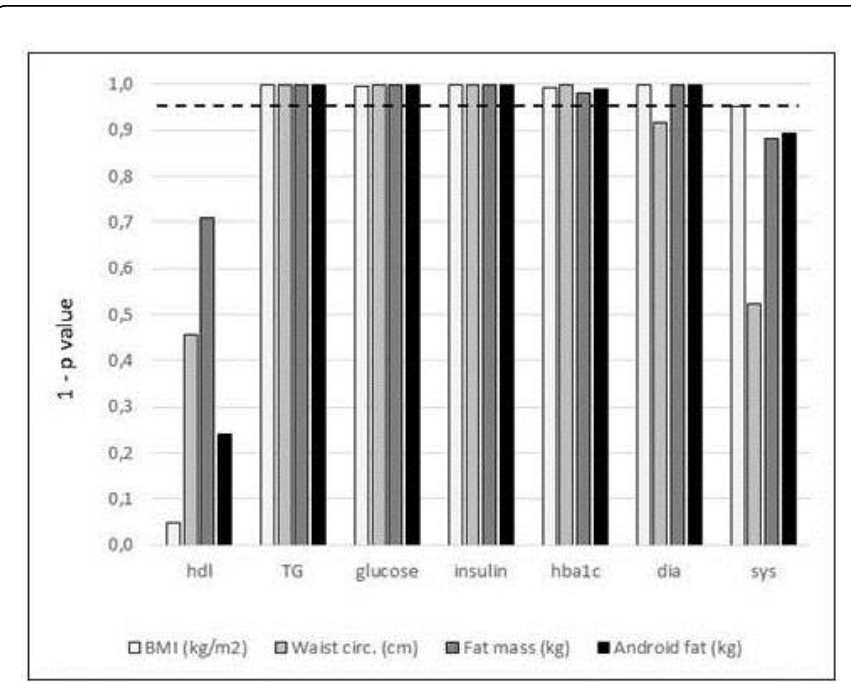

Figure 1: Associations between body fat estimates and cardiovascular risk factors in male, the columns represent 1-p-value according to a linear regression model corrected for age-the dotted line in the figure indicates the $5 \%$ significance.

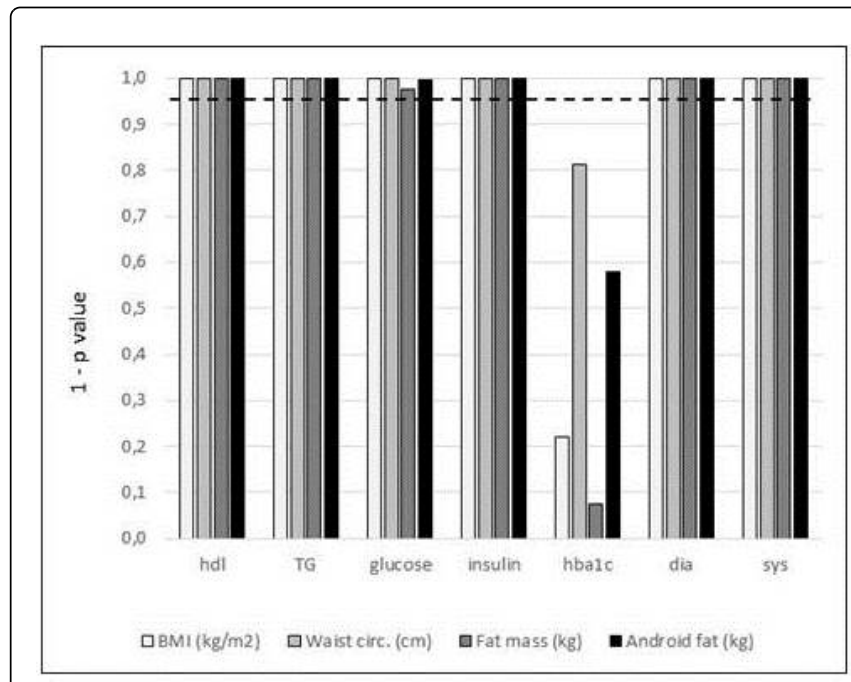

Figure 2: Associations between body fat estimates and cardiovascular risk factors in female, the columns represent 1 - $\mathrm{p}$ value according to a linear regression model corrected for age-the dotted line in the figure indicates the $5 \%$ significance.

The columns represent 1-p-value from the corresponding linear regression model. A higher column means a higher statistical association. The dotted line in the figure indicates the $5 \%$ significance level. A very similar pattern is seen for all the body fat estimates. However there were some gender differences. Body fatness was not related to TC or LDL (not shown).

ROC curves showed that a BMI $\geq 25$ is reasonably sensitive to identify participants with risk factors outside the normal range. Sensitivity is shown for men and women in brackets, respectively: HDL cholesterol (91\%; $81 \%)$, triglycerides $(89 \%$; $70 \%)$, fasting glucose $(88 \%$; $84 \%)$, fasting insulin $(100 \% ; 80 \%)$ and hypertension $(83 \% ; 84 \%)$. ROC 
curves were not used for $\mathrm{HbAlC}$, because only very few participants were above the threshold of $7 \%$.

\section{Discussion}

The current study investigated the associations between estimates of body fatness and cardiovascular risk factors in community dwelling old adults. Contrary to our expectations, we found that correlations between BMI and other body fat estimates were constant in both genders throughout age tertiles, and further on, we found that fat estimates derived from DXA did not show stronger associations with cardiovascular risk than simple BMI.

Body composition usually changes during ageing mainly due to an increase in fat mass and a decrease in lean body mass in old age groups [18]. In our participants, age was related to lower BMI but not to lower body fat percentage (BF\%). It has to be considered that most of our participants were between 70 and 80 years old. This narrow age distribution makes is difficult to observe the expected increase in BF\% because it has been reported that these changes start earlier in life [19]. Additionally, our participants were volunteers in an exercise intervention program [17], thus they were physically active and fit. It is reasonable to assume that in physically active people adverse changes in body composition proceed slower compared to physically inactive peers [20].

In general, our study confirmed the positive relationship between BMI and BF\%. This has been shown in previous studies $[20,22,23]$. However, it was unexpected that the associations between BMI and BF $\%$ were similar in all age tertiles. It is known that $\mathrm{BF} \%$ is not just a function of BMI, but also of age $[21,22]$. Thus, we expected that due to age related changes in body composition, BMI would show a lesser association with $\mathrm{BF} \%$ in the oldest age tertile. However, this was not the case. It has been shown that the BMI/BF\% relation is stronger at higher BMI and weaker in the BMI range $20-25 \mathrm{~kg} / \mathrm{m} 2$ [19]. Our participants were mostly in the overweight and obesity category thus explaining this finding.

We measured clinical risk factors in blood of the participants and additionally blood pressure. All of them have been shown to predict future cardiovascular morbidity and/or mortality [23-26]. In our study we found that the different estimates of body fatness were associated with cardiovascular risk factors in a very similar way. It has been shown previously that overweight and obesity are related to poorer lipid- and glucose metabolism as well as to hypertension [27-29].

However, it was somehow unexpected that measures derived from DXA, in particularly visceral fat, did not show a stronger association with cardiovascular risk than BMI. Many epidemiologic studies have indicated that visceral fat is a major risk factor for insulin resistance, type 2 diabetes mellitus, cardiovascular disease, stroke, metabolic syndrome and death [30]. It has even been associated with cardiovascular risk in young and lean individuals [31].

Visceral fat has been discussed to be linked causally to metabolic disease. The mechanism is thought to involve its anatomical location, leading to a 'portal' effect of greater free fatty acids (FFA) and glycerol release. Evidence has shown that adipose tissue is an active endocrine organ, capable of secreting many cytokines, often referred to as adipokines, which can promote inflammation and interfere with insulin action $[28,32,33]$. However, our study indicates that BMI is (statistically seen) equally related to cardiovascular risk factors, probably due to its own associations with visceral fat.
Interestingly, we could not find associations between body fatness and total cholesterol and LDL although this has been reported previously [34]. For some risk factor, we found that their correlations with body fat were different between genders. In our study, TG, glucose, insulin and DBP showed similar associations in both genders, but HDL and SBP were associated with body fatness in women only whereas HbA1c was associated with body fatness in men only. The metabolic response to obesity differs between sexes potentially explaining why obesity-related cardiovascular mortality is lower in women than in men at every BMI level $[35,36]$.

Although it has been shown that overweight and obese individuals can be metabolically healthy, the observed associations between body fatness and risk factors in our study indicate that risk increases with increasing body fatness in old adults. Although there are obvious gender differences how lipid metabolism, glucose metabolism and blood pressure are associated to body fatness, it might be of clinical relevance that, according to our data, no potentially protective effect of high body fatness was observed in our participants and that this was independent from what body fat estimate was used. Considering the simplicity of BMI and its sensitivity to detect people at cardiovascular risk, routine use of BMI during clinical assessment is encouraged.

\section{Limitations}

The current data analysis was based on a cross-sectional data set and it is in the nature of this kind of study that observed associations neither proof causality nor determine the direction of an association. Further, our participants were volunteers in an exercise intervention program [37], thus they were physically active and fit and not necessarily representative for this age group. Thus, it is not known whether the results are valid for other population groups.

\section{Conclusion}

Our results indicate that BMI is highly related to body fatness measured by DXA in old adults and that DXA assessment of body composition is not necessarily superior in estimating cardiovascular risk compared to simple BMI. The results also indicate that risk factors are associated differently with body fatness in men and women. Further, BMI seems to be a suitable and simple tool to identify subjects at cardiovascular risk even at old age.

\section{Acknowledgment}

All authors are responsible for the reported research and declare that they have no competing interests. This study was part of the IceProQualita project, which was funded by the Icelandic Technology Development Fund (No 071323008), Research Fund of the University of Iceland, a grant from Landspitali University Hospital Research Fund and the Helga Jonsdottir and Sigurlidi Kristjansson Geriatric Research Fund. The trial is registered at the US National Library of Medicine (No. NCT01074879).

\section{Take away Points}

- BMI is related to body fatness in old men and women.

- Simple BMI is equally related to cardiovascular risk as is expensive DXA measurements.

- BMI is a suitable tool to identify subjects at cardiovascular risk even at old age. 


\section{References}

1. Bauer UE, Briss PA, Goodman RA, Bowman BA (2014) Prevention of chronic disease in the 21st century: elimination of the leading preventable causes of premature death and disability in the USA. Lancet 384: 45-52.

2. Cerhan JR, Moore SC, Jacobs EJ, Kitahara CM, Rosenberg PS, et al. (2014) A pooled analysis of waist circumference and mortality in 650,000 adults. Mayo Clin Proc 89: 335-345.

3. Deglise C, Bouchardy C, Burri M, Usel M, Neyroud-Caspar I, et al. (2010) Impact of obesity on diagnosis and treatment of breast cancer. Breast Cancer Res Treat 120: 185-193.

4. Martín-Timón I (2014) Type 2 diabetes and cardiovascular disease: Have all risk factors the same strength? World J Diabetes 5: 444-470.

5. Koppe SW (2014) Obesity and the liver: nonalcoholic fatty liver disease. Transl Res 164: 312-322.

6. Acosta A and Camilleri M (2014) gastrointestinal morbidity in obesity. Ann N Y Acad Sci 1311: 42-56.

7. World Health organization (Updated October 2017) Obesity and overweight. Fact sheet $\mathrm{N}^{\circ} 311$.

8. Huxley R, Mendis S, Zheleznyakov E, Reddy S, Chan J (2009) Body mass index, waist circumference and waist: hip ratio as predictors of cardiovascular risk-a review of the literature. Eur J Clin Nutr 64: 16-22.

9. Bastien M, Poirier P, Lemieux I, Després JP (2014) Overview of epidemiology and contribution of obesity to cardiovascular disease. Prog Cardiovasc Dis 56: 369-381.

10. Chapman IM (2010) Obesity paradox during aging. Interdiscip Top Gerontol 37: 20-36.

11. Goel K, Lopez-Jimenez F, De Schutter A, Coutinho T, Lavie CJ (2014) Obesity paradox in different populations: evidence and controversies. Future Cardiol 10: 81-91.

12. Cheng FW, Gao X, Mitchell DC, Wood C, Rolston DD, et al. (2016) Metabolic Health Status and the Obesity Paradox in Older Adults. J Nutr Gerontol Geriatr 35: 161-176.

13. Brady AO, Straight CR, Evans EM (2014) Body composition, muscle capacity, and physical function in older adults: an integrated conceptual model. J Aging Phys Act 22: 441-452.

14. Kim TN and Choi KM (2013) Sarcopenia: definition, epidemiology, and pathophysiology. J Bone Metab 20: 1-10.

15. Barry VW, Baruth M, Beets MW, Durstine JL, Liu J, et al. (2014) Fitness vs. fatness on all-cause mortality: a meta-analysis. Prog Cardiovasc Dis 56: 382-390.

16. Ströhle A, Worm N (2014) [Healthy obesity? Why the adiposity paradox is only seemingly paradox]. [Article in German] Med Monatsschr Pharm 37: 54-64.

17. Arnarson A, Geirsdottir OG, Ramel A, Briem K, Jonsson PV, et al. (2013) Effects of whey proteins and carbohydrates on the efficacy of resistance training in elderly people: double blind, randomised controlled trial. EJCN 67: 821-826.

18. Folstein MF, Folstein SE, McHugh PR (1975) "Mini-mental state". A practical method for grading the cognitive state of patients for the clinician. J Psychiatr Res 12: 189-198.

19. Meeuwsen S, Horgan GW, Elia M (2010) The relationship between BMI and percent body fat, measured by bioelectrical impedance, in a large adult sample is curvilinear and influenced by age and sex. Clin Nutr 29: 560-566.

20. Strugnell C, Dunstan DW, Magliano DJ, Zimmet PZ, Shaw JE, et al. (2014) Influence of age and gender on fat mass, fat-free mass and skeletal muscle mass among Australian adults: the Australian diabetes, obesity and lifestyle study (AusDiab). J Nutr Health Aging 18: 540-546.

21. Landi F, Marzetti E, Martone AM, Bernabei R, Onder G (2014) Exercise as a remedy for sarcopenia. Curr Opin Clin Nutr Metab Care 17: 25-31.

22. Gallagher D, Visser M, Sepúlveda D, Pierson RN, Harris T, et al. 81996) How useful is body mass index for comparison of body fatness across age, sex, and ethnic groups? Am J Epidemiol 143: 228-239.

23. Jackson AS, Stanforth PR, Gagnon J, Rankinen T, Leon AS, et al. (2002) The effect of sex, age and race on estimating percentage body fat from body mass index: The Heritage Family Study. Int J Obes Relat Metab Disord 26: 789-796.

24. Wang Y, Lammi-Keefe CJ, Hou L, Hu G (2013) Impact of low-density lipoprotein cholesterol on cardiovascular outcomes in people with type 2 diabetes: a meta-analysis of prospective cohort studies. Diabetes Res Clin Pract 102: 65-75.

25. Boekholdt SM, Arsenault BJ, Hovingh GK, Mora S, Pedersen TR, et al. (2013) Levels and changes of HDL cholesterol and apolipoprotein A-I in relation to risk of cardiovascular events among statin-treated patients: a meta-analysis. Circulation 128: 1504-1512.

26. Erqou S, Lee CT, Suffoletto M, Echouffo-Tcheugui JB, de Boer RA, et al. (2013) Association between glycated haemoglobin and the risk of congestive heart failure in diabetes mellitus: systematic review and metaanalysis. Eur J Heart Fail 15: 185-193.

27. Asayama K, Satoh M, Murakami Y, Ohkubo T, Nagasawa SY, et al. (2014) Cardiovascular risk with and without antihypertensive drug treatment in the Japanese general population: participant-level meta-analysis. Hypertension 63: 1189-1197.

28. Dorresteijn JA, Visseren FL, Spiering W (2012) Mechanisms linking obesity to hypertension. Obes Rev 13: 17-26.

29. Krauss RM, Winston M, Fletcher BJ, Grundy SM (1998) Obesity: impact on cardiovascular disease Circulation 98: 1472-1476.

30. Castro AV, Kolka CM, Kim SP, Bergman RN (2014) Obesity, insulin resistance and comorbidities? Mechanisms of association. Arq Bras Endocrinol Metabol 58: 600-609.

31. Finelli C, Sommella L, Gioia S, La Sala N, Tarantino G (2013) Should visceral fat be reduced to increase longevity? Ageing Res Rev 12: 996-1004.

32. Miazgowski T, Krzyżanowska-Świniarska B, Dziwura-Ogonowska J, Widecka K (2014) The associations between cardiometabolic risk factors and visceral fat measured by a new dual-energy X-ray absorptiometryderived method in lean healthy Caucasian women. Endocrine 47: 500-505.

33. Harwood HJ (2012) The adipocyte as an endocrine organ in the regulation of metabolic homeostasis. Neuropharmacology 63: 57-75.

34. Cohen-Mansfield J, Perach R (2011) Is there a reversal in the effect of obesity on mortality in old age? J Aging Res 1-8.

35. Cong L, Zhan JQ, Yang L, Zhang W, Li SG, et al. (2014) Overweight and obesity among low-income Muslim Uyghur women in far western China: correlations of body mass index with blood lipids and implications in preventive public health. PLoS One 9: e90262.

36. Thomas F, Bean K, Pannier B, Oppert JM, Guize L, et al. (2005) Cardiovascular mortality in overweight subjects: the key role of associated risk factors. Hypertension 46: 654-659.

37. Berrington de Gonzalez A, Hartge P, Cerhan JR, Flint AJ, Hannan L, et al. (2011) Body-mass index and mortality among 1.46 million white adults. N Engl J Med 363: 2211-2219. 